\title{
Brimonidine prevents neurodegeneration in a mouse model of normal tension glaucoma
}

\author{
K Semba ${ }^{1,2}$, K Namekata $^{1}$, A Kimura ${ }^{1}$, C Harada ${ }^{1}$, Y Mitamura $^{2}$ and T Harada ${ }^{*, 1,2}$
}

Glaucoma is one of the leading causes of irreversible blindness that is characterized by progressive degeneration of optic nerves and retinal ganglion cells (RGCs). In the mammalian retina, excitatory amino-acid carrier 1 (EAAC1) is expressed in neural cells, including RGCs, and the loss of EAAC1 leads to RGC degeneration without elevated intraocular pressure (IOP). Brimonidine (BMD) is an $\alpha 2$-adrenergic receptor agonist and it is commonly used in a form of eye drops to lower IOP in glaucoma patients. Recent studies have suggested that BMD has direct protective effects on RGCs involving IOP-independent mechanisms, but it is still controversial. In the present study, we examined the effects of BMD in EAAC1-deficient (KO) mice, an animal model of normal tension glaucoma. BMD caused a small decrease in IOP, but sequential in vivo retinal imaging and electrophysiological analysis revealed that treatment with BMD was highly effective for RGC protection in EAAC1 KO mice. BMD suppressed the phosphorylation of the $\mathrm{N}$-methyl-D-aspartate receptor 2B (NR2B) subunit in RGCs in EAAC1 KO mice. Furthermore, in cultured Müller glia, BMD stimulated the production of several neurotrophic factors that enhance RGC survival. These results suggest that, in addition to lowering IOP, BMD prevents glaucomatous retinal degeneration by stimulating multiple pathways including glia-neuron interactions.

Cell Death and Disease (2014) 5, e1341; doi:10.1038/cddis.2014.306; published online 17 July 2014

Glaucoma is one of the leading causes of vision loss in the world. It is estimated that glaucoma will affect more than 80 million individuals worldwide by 2020 , with at least $6-8$ million individuals becoming bilaterally blind. $^{1}$ The disease is characterized by the progressive degeneration of retinal ganglion cells (RGCs) and their axons, which are usually associated with elevated intraocular pressure (IOP). On the other hand, normal tension glaucoma (NTG) is a subtype of glaucoma that presents with statistically normal IOP. The prevalence of NTG is reported to be higher among the Japanese than among Caucasians. ${ }^{2}$ These findings suggest a possibility that non-IOP-dependent factors may contribute to disease progression of glaucoma, especially in the context of NTG. ${ }^{3,4}$ For example, an excessively high extracellular concentration of glutamate chronically activates glutamate receptors, such as $N$-methyl-D-aspartate (NMDA) receptors, and allows calcium entry into the cell causing an uncontrolled elevation of intracellular calcium levels. This process is thought to be one of the causes of RGC death. ${ }^{3-5}$ The glutamate transporter (GLT) is the only mechanism for removal of glutamate from the extracellular fluid in the retina. ${ }^{3,6,7}$ In the inner plexiform layer where synapses exist across RGCs, at least three transporters are involved in this task: GLT-1 located in the bipolar cell terminals; excitatory amino-acid carrier 1 (EAAC1) in RGCs; and glutamate/aspartate transporter (GLAST) in Müller glial cells. ${ }^{3,7,8}$ We previously reported that EAAC1 and GLAST knockout (KO) mice show progressive RGC loss and optic nerve degeneration without elevated IOP, and not only glutamate neurotoxicity but also oxidative stress is involved in its mechanism. ${ }^{3,8-10}$ In adult EAAC1 and GLAST KO mice, lipid hydroperoxides were increased and glutathione concentrations were decreased in retinas, suggesting the involvement of oxidative stress in RGC loss. In addition, cultured RGCs prepared from EAAC1 KO mice were more vulnerable to oxidative stress. ${ }^{3}$ Oxidative stress has been proposed to contribute to retinal damage in various eye diseases including glaucoma and age-related macular degeneration. ${ }^{11,12}$ Taken together with the downregulation of GLTs and glutathione levels observed in glaucoma patients, ${ }^{13}$ these mice seem to be useful as the animal models of NTG.

Brimonidine (BMD) is a selective $\alpha 2$-adrenergic receptor agonist that lowers IOP by reducing the production of aqueous humor and facilitating its exit via the trabecular meshwork. ${ }^{14}$ Recent studies have shown that BMD protects RGCs from glutamate neurotoxicity, oxidative stress and hypoxia in vitro. ${ }^{15,16}$ In addition, BMD provides neuroprotective effects in various animal models of optic neuropathy including experimental glaucoma, ischemia, oxidative stress and optic nerve injury. ${ }^{17-19}$ BMD may exert its neuroprotective effects via the upregulation of neurotrophic factors, such as brainderived neurotrophic factor (BDNF) ${ }^{20}$ and basic fibroblast growth factor (bFGF), ${ }^{21,22}$ in RGCs. Thus, the neuroprotective

\footnotetext{
${ }^{1}$ Visual Research Project, Tokyo Metropolitan Institute of Medical Science, Tokyo, Japan and ${ }^{2}$ Department of Ophthalmology, Institute of Health Biosciences, The University of Tokushima Graduate School, Tokushima, Japan

*Corresponding author: T Harada, Visual Research Project, Tokyo Metropolitan Institute of Medical Science, 2-1-6 Kamikitazawa, Setagaya-ku, Tokyo 156-8506, Japan. Tel: +81 36834 2338; Fax: +81 36834 2339; E-mail: harada-tk@igakuken.or.jp

Abbreviations: BDNF, brain-derived neurotrophic factor; bFGF, basic fibroblast growth factor; BMD, brimonidine; EAAC1, excitatory amino-acid carrier 1; GCL, ganglion cell layer; GFAP, glial fibrillary acidic protein; GLAST, glutamate/aspartate transporter; IOP, intraocular pressure; mfERG, multifocal electroretinogram; NMDA, $\mathrm{N}$-methyl-D-aspartate; OCT, optical coherence tomography; RGC, retinal ganglion cell

Received 20.5.14; accepted 09.6.14; Edited by A Verkhratsky
} 
effects of BMD seem to be, at least partly, through IOPindependent factors, but the detailed mechanism are still unknown. Fujita et al. ${ }^{23}$ recently reported that topical administration of BMD promotes axon regeneration after optic nerve injury. BMD increased the expression of the tropomyosin receptor kinase $B$ (TrkB), a high-affinity $B D N F$ receptor, in the mouse retina. We previously reported that BDNF-TrkB signaling in Müller glial cells have important roles in the production of trophic factors including BDNF and bFGF, and in the protection from glutamate-induced RGC death and druginduced photoreceptor death. ${ }^{24}$ Systemically administered $\alpha 2$-adrenergic agonists are known to activate selectively extracellular signal-regulated kinases in Müller cells in vivo. ${ }^{25}$ These results suggest a possibility that BMD may stimulate the production of trophic factors in not only RGCs but also in Müller cells. In the present study, we show that BMD prevents glaucomatous retinal degeneration in EAAC1 KO mice, an animal model of NTG, and we report novel IOPindependent pathways for BMD-mediated neuroprotection that involve NMDA receptors and glia-neuron interaction.

\section{Results}

BMD protects RGCs in EAAC1 KO mice. To investigate whether BMD is capable of preventing the NTG-like phenotypes in EAAC1 KO mice, we administered BMD or PBS (control) locally every day to EAAC1 KO mice from 5 weeks old $(5 \mathrm{~W})$ to $8 \mathrm{~W}$ (Figure 1a). The retinas of EAAC1 KO mice show normal organization at $5 \mathrm{~W}$, but RGC loss and the thinning in the inner retina was clear at $8 \mathrm{~W}$ (Figure 1b). ${ }^{3,8,10}$ The cell number in the ganglion cell layer (GCL) at $8 \mathrm{~W}$ was significantly lower in EAAC1 KO mice $(356 \pm 3 ; n=6)$ compared with WT mice $(448 \pm 8 ; n=6)$ (Figure 1c). In addition, the thickness of the inner retinal layer (IRL) was significantly decreased at $8 \mathrm{~W}$ in EAAC1 $\mathrm{KO}$ mice $(100 \pm 2 \mu \mathrm{m} ; n=6)$ (Figure 1d). In BMD-treated EAAC1 KO mice, the number of surviving neurons was significantly higher $(396 \pm 3 ; n=6)$ than that in control EAAC1 KO mice (Figures $1 \mathrm{~b}$ and $\mathrm{c}$ ). In addition, BMD treatment prevented the thinning of the IRL $(108 \pm 2 \mu \mathrm{m} ; n=6)$ (Figure $1 \mathrm{~d})$. We also visualized retinal layers using optical coherence tomography

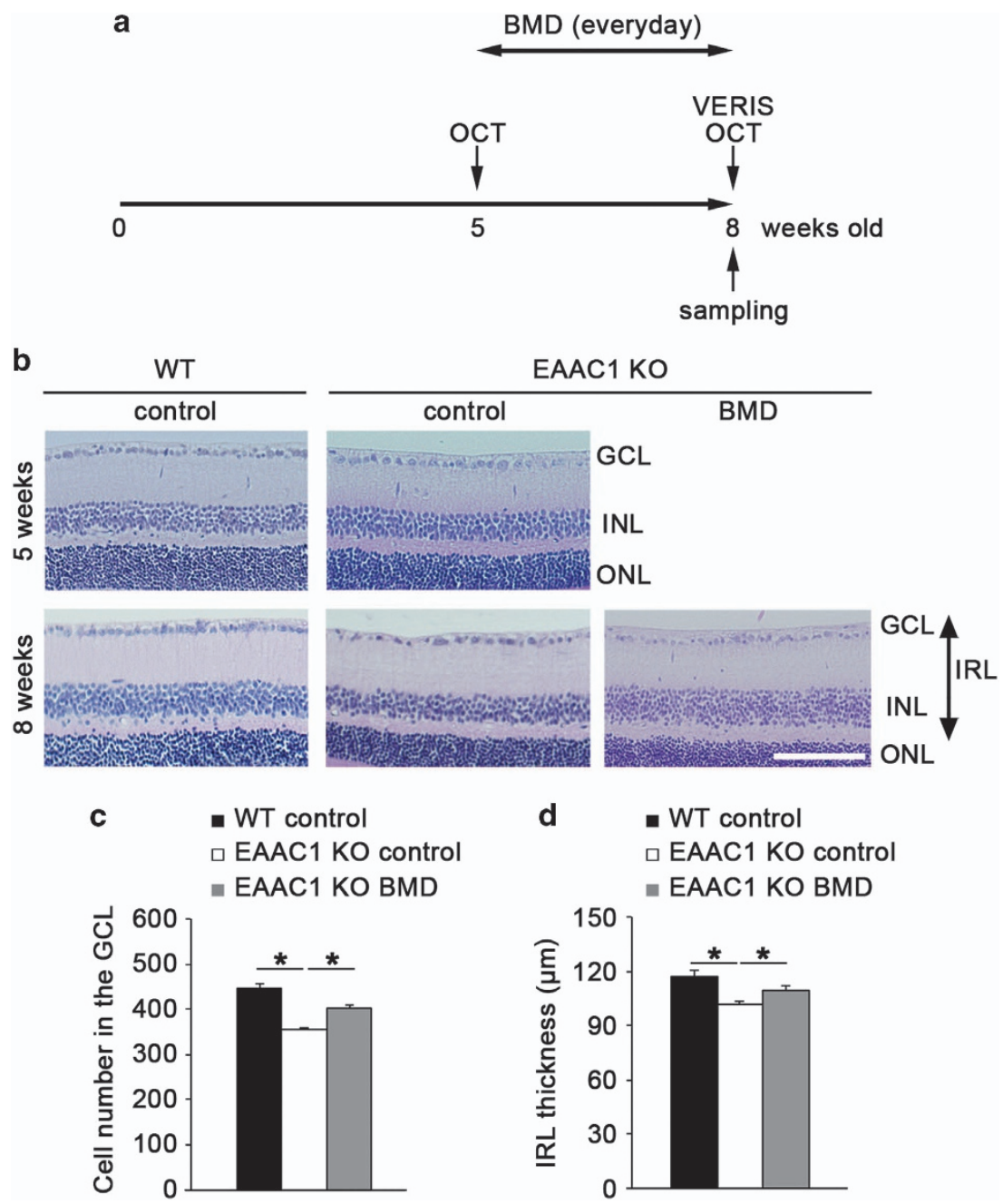

Figure 1 Effects of BMD on RGC loss and IRL thickness in EAAC1 KO mice. (a) Animal protocols. 1.0\% BMD or PBS (5 $\mu$ ) was administered locally every day from $5 \mathrm{~W}$ to $8 W$. The mice were killed at $5 W$ and $8 W$. (b) H\&E staining of retinal sections at $5 W$ and $8 W$ in WT and EAAC1 KO mice. Scale bar: $100 \mu$ m. INL, inner nuclear layer; . (c and d) Quantification of the RGC number (c) and IRL thickness (d). The data are presented as means \pm S.E.M. of six samples for each experiment. ${ }^{\star} P<0.05$ 
(OCT), a noninvasive imaging technique that can be used to acquire cross-sectional tomographic images of the retina in vivo. ${ }^{10,26}$ The average thickness of the ganglion cell complex (GCC), which includes the nerve fiber layer, GCL, and inner plexiform layer, was significantly greater in BMD-treated EAAC1 KO mice $(99 \pm 1 \% ; n=6)$ compared with control EAAC1 KO mice ( $88 \pm 1 \% ; n=6)$ (Figure 2). These data indicate that BMD treatment protects RGCs from NTG-like neurodegeneration.

BMD improves visual function in EAAC1 KO mice. To determine whether the observed BMD-induced RGC protection is functionally significant, we analyzed multifocal electroretinograms (mfERGs) of EAAC1 KO mouse retinas. Functional RGCs contribute to eliciting the mfERG response, and the second-order kernel $(2 \mathrm{~K})$, which appears to be a sensitive indicator of inner retinal dysfunction, ${ }^{27}$ is impaired in glaucoma patients. ${ }^{28}$ The averaged $2 \mathrm{~K}$ responses of six mice are shown in Figure $3 a$. The response topography demonstrated that the $2 \mathrm{~K}$ component derived from EAAC1 $\mathrm{KO}$ mice tended to be smaller in all visual fields compared with that from WT mice. Quantitative analysis revealed that the $2 \mathrm{~K}$ amplitude was reduced in EAAC1 $\mathrm{KO}$ mice $\left(2.4 \pm 0.1 \mathrm{nV} / \mathrm{deg}^{2} ; n=6\right)$ compared with that in WT mice $\left(3.8 \pm 0.1 \mathrm{nV} / \mathrm{deg}^{2} ; n=6\right)$ (Figure 3b). However, BMD prevented the reduction of the $2 \mathrm{~K}$ amplitude in EAAC1 $\mathrm{KO}$ mice $\left(3.0 \pm 0.2 \mathrm{nV} / \mathrm{deg}^{2} ; n=6\right)$, suggesting that the neuroprotective effect of $B M D$ is functionally significant in EAAC1 KO mice.

Effects of BMD on IOP in EAAC1 KO mice. IOP measurement was carried out at $5 \mathrm{~W}$ and $8 \mathrm{~W}$ mice at around 2100 hours, when IOP is the highest in mouse eyes. $^{29}$ The IOP of EAAC1 KO mice was not significantly increased compared with WT at both $5 \mathrm{~W}$ and $8 \mathrm{~W}$ (Figure 4). BMD slightly, but significantly, reduced IOP $(10.8 \pm 0.6 \mathrm{mmHg} ; n=6)$ compared with control EAAC1 $\mathrm{KO}$ mice $(13.1 \pm 0.3 \mathrm{mmHg} ; n=6)$. These results suggest that BMD-induced RGC protection may, at least partly, involve an IOP-dependent mechanism.

BMD reduces phosphorylated NR2B expression in EAAC1 KO mice. We recently reported that inactivation of NR2B, one of the NMDA receptor subunits, protects RGCs from glutamate neurotoxicity. ${ }^{5}$ Since BMD exerts direct neuroprotective effects on RGCs, ${ }^{15,16}$ BMD may affect the NMDA receptor activity in the retina. To determine this possibility, we next examined total and phosphorylated (at Tyr1472) NR2B protein expressions in EAAC1 KO mice before and after BMD treatment. The ratio of phosphorylated/ total NR2B in EAAC1 KO mice was significantly higher compared with that in WT mice $(161 \pm 13 \% ; n=6)$, but such an increased level was not detected after BMD treatment $(123 \pm 12 \% ; n=6)$ (Figure 5a). Immunohistochemical analysis revealed that immunoreactivity of phosphorylated NR2B was low in WT mice $(100 \pm 11 \% ; n=6)$, but it was significantly increased, especially in RGCs, in EAAC1 KO mice $(167 \pm 14 \% ; n=6)$ at $8 \mathrm{~W}$ (Figures $5 \mathrm{~b}$ and $\mathrm{c}$ ), suggesting an excitotoxic environment in EAAC1 $\mathrm{KO}$ mice. The elevation of the phosphorylated NR2B expression was significantly
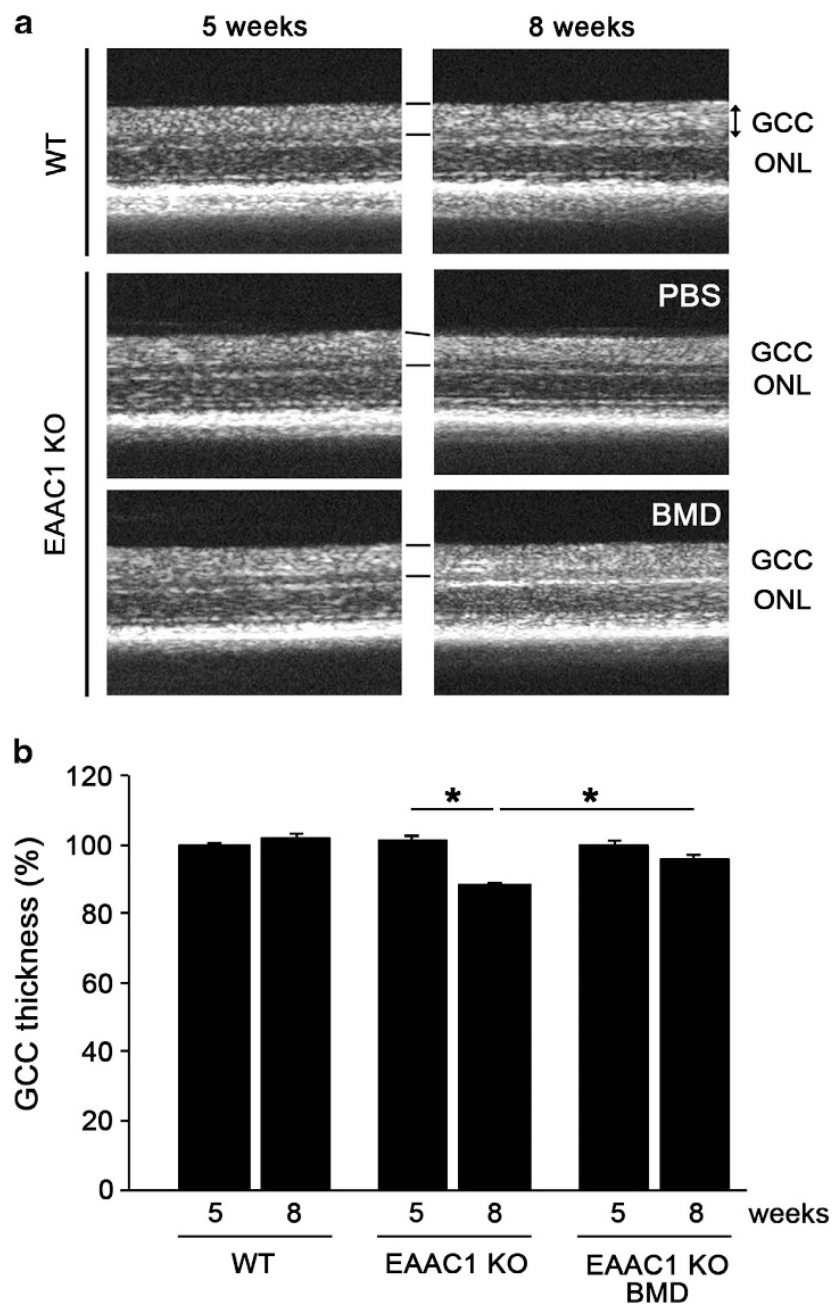

Figure 2 In vivo imaging of the retina in EAAC1 $\mathrm{KO}$ mice treated with BMD. (a) OCT cross-sectional images of retinas at $5 \mathrm{~W}$ and $8 \mathrm{~W}$. (b) Longitudinal evaluation of the GCC thickness. The data are presented as means \pm S.E.M. of six samples for each experiment. ${ }^{*} P<0.05$

suppressed by BMD treatment $(122 \pm 10 \% ; n=6)$. These results suggest that BMD exerts a direct neuroprotective effect, at least partly, by reducing the activated NMDA receptor expression and thus suppressing excitotoxicity. ${ }^{15-18}$

BMD stimulates trophic factor productions in Müller glial cells. The cross-talk between glia and neurons in neuroprotection is now well known, and during retinal degeneration, Müller glial cells produce several trophic factors and rescue retinal neurons. ${ }^{30,31}$ Thus, we next examined whether BMD stimulates trophic factor production in cultured Müller cells prepared from WT mice. BMD significantly increased mRNA expression levels of the nerve growth factor (NGF; $186 \pm 12 \% ; n=6)$, BDNF $(441 \pm 18 \% ; n=6)$ and bFGF $(265 \pm 8 \% ; n=6)$, but not of neurotrophin-3 (NT-3), ciliary neurotrophic factor (CNTF) and glial cell line-derived neurotrophic factor (GDNF) (Figure 6). These results suggest that BMD has the capacity to stimulate the production of several trophic factors in Müller cells, and these trophic factors may act on RGCs leading to RGC protection indirectly. ${ }^{24}$ 
a

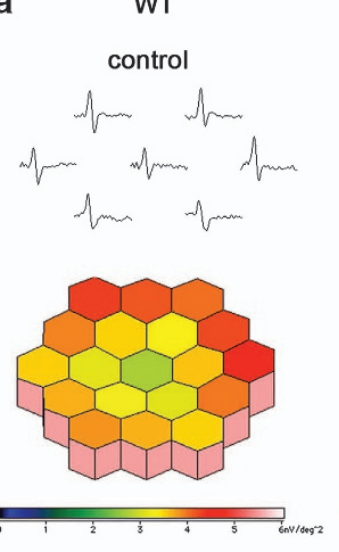

EAAC1 KO

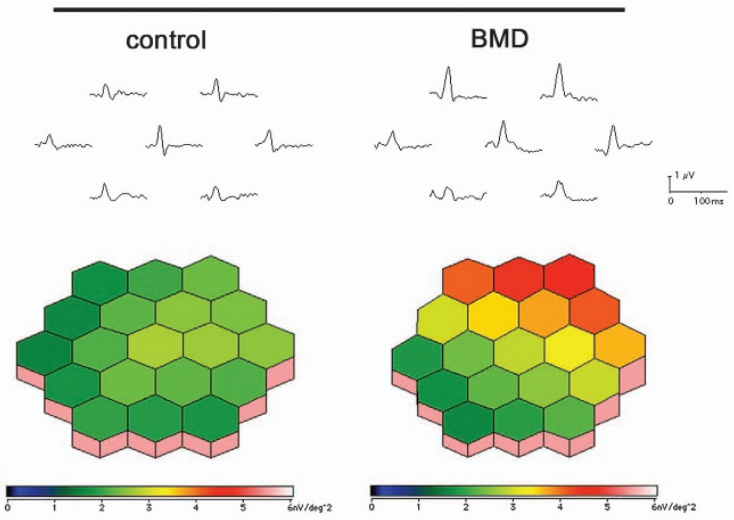

b

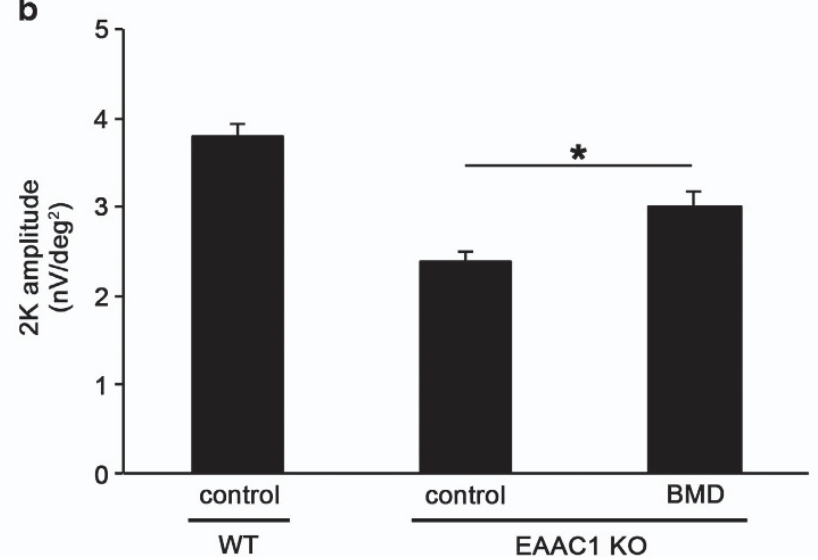

Figure 3 Effects of BMD on visual responses in EAAC1 KO mice. (a) Averaged visual responses of the second-order kernel (2K) demonstrated using 3D plots. (b) Quantitative analysis of the visual response amplitude. The data are presented as means \pm S.E.M. of six samples for each experiment. ${ }^{*} P<0.05$

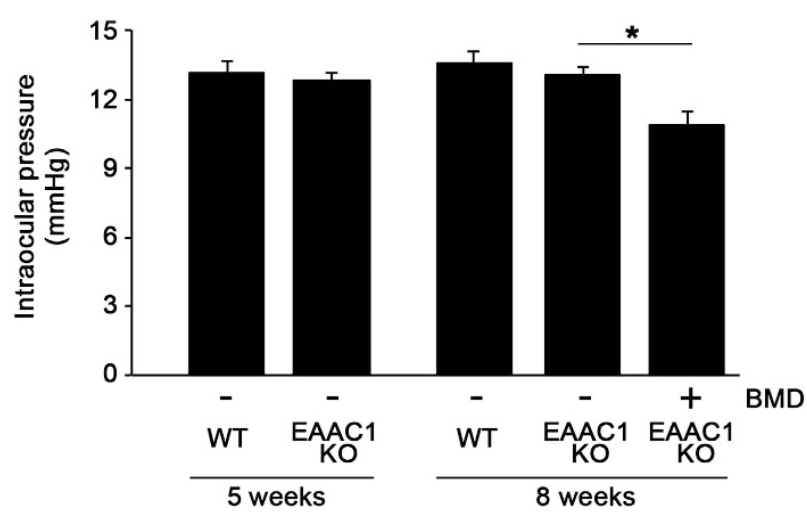

Figure 4 Effects of BMD on IOP in EAAC1 KO mice. The data are presented as means \pm S.E.M. of six samples for each experiment. ${ }^{*} P<0.05$

\section{Discussion}

This was the first study to investigate the neuroprotective effects of BMD in a mouse model of NTG. We showed that BMD prevents progressive RGC loss, thinning of the IRL and visual impairment in EAAC1 KO mice. To demonstrate these findings in the same animal, we used OCT and mfERG that permit in vivo, noninvasive, longitudinal and quantitative assessment of the changes in retinal morphology and function in EAAC1 KO mice. These techniques clearly visualized the neuroprotective effects of BMD in the present study and they are useful for providing information in experimental animals as well as in clinical trials and management. ${ }^{32}$ The IOP of EAAC1 $\mathrm{KO}$ mice are low and BMD slightly decreased the IOP of these mice, suggesting that the clear neuroprotective effects of BMD are mostly independent of its ability to lower IOP. This concept supports the findings from a recent study that reported low-pressure glaucoma patients treated with BMD $0.2 \%$ are less likely to have field progression than patients treated with timolol $0.5 \% .{ }^{33}$ As the mean IOP was similar in both groups at the 4-year follow-up, these results suggested that BMD has IOP-independent neuroprotective effects on RGCs. Our data suggest that one of the IOP-independent mechanisms is suppression of excitotoxicity in RGCs. Excitotoxicity is implicated in the degeneration of RGCs and optic nerves observed under pathologic conditions including glaucoma. ${ }^{5,34}$ In addition, overactivation of NMDA receptors is thought to be a key contributing factor in the pathophysiology of many central nervous system disorders, such as Alzheimer's disease ${ }^{35}$ and Huntington disease. ${ }^{36}$ In the EAAC1 KO mouse retina, the phosphorylated NR2B expression level was upregulated, and BMD significantly inhibited this effect, suggesting that excitotoxicity is reduced in BMDtreated EAAC1 $\mathrm{KO}$ mice leading to increased RGC survival. Another IOP-independent mechanism may be stimulation of 
a

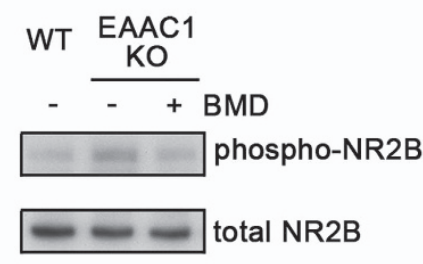

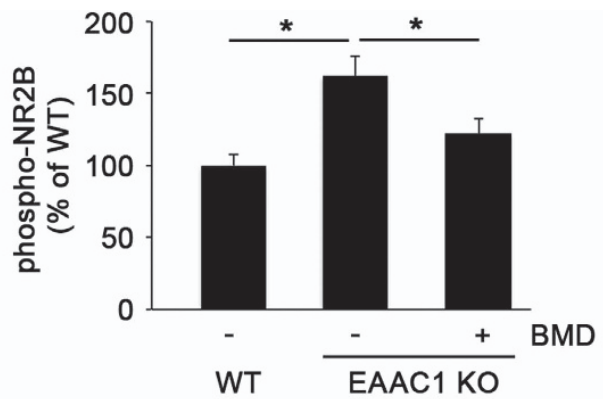

b
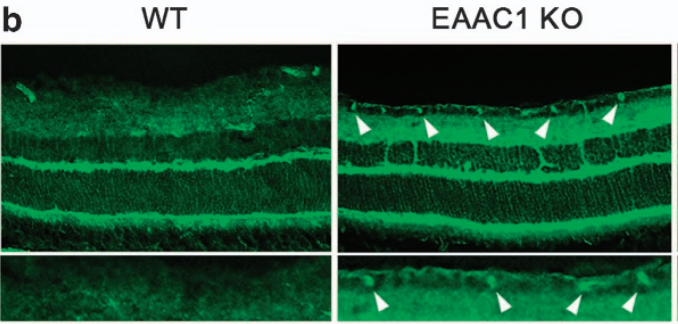

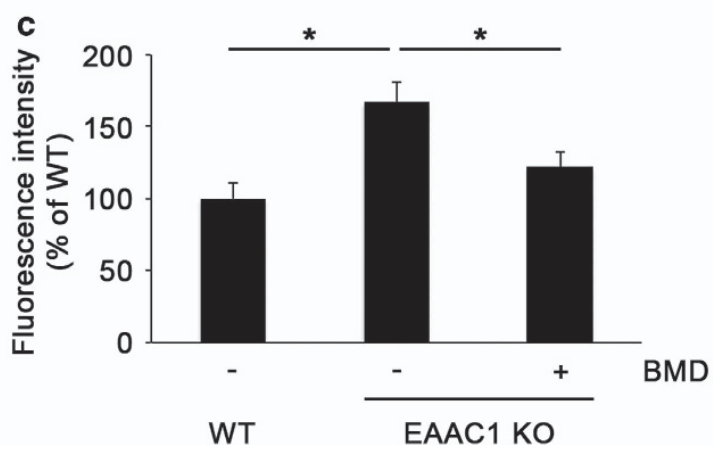

Figure 5 Effects of BMD on phosphorylation of NR2B in the retina. (a) Immunoblot analysis of phosphorylated and total NR2B in the retinas of WT, EAAC1 KO and BMDtreated EAAC1 KO mice. Ratio of NR2B phosphorylation in WT mice was estimated as $100 \%$. (b) Immunohistochemical analysis of mouse retinas stained with an antibody against phosphorylated NR2B in WT, EAAC1 KO and BMD-treated EAAC1 KO mice. Arrowheads indicate RGCs. INL, inner nuclear layer. Scale bar: 100 and $50 \mu \mathrm{m}$ in upper and lower rows, respectively. (c) Quantitative analysis of the phosphorylated NR2B intensity in (b). The NR2B fluorescence intensity in WT mice was estimated as $100 \%$. The data are presented as means \pm S.E.M. of six samples for each experiment. ${ }^{*} P<0.05$

glia-neuron interactions. Previous studies have shown that BMD directly increases BDNF and bFGF production in RGCs. ${ }^{15,16,21,22}$ However, production of neurotrophic factors in Müller glial cells has not been investigated previously. We found that BMD stimulates the production of NGF, BDNF and bFGF, all of which induce RGC survival, in Müller glial cells. $^{24,37}$ Taken together, in addition to lowering IOP, BMD may prevent glaucomatous retinal degeneration by stimulating multiple pathways including glia-neuron interactions.

Recent studies have reported a possibility that blockade of axonal transport in glaucoma leads to deficits in the neurotrophic factors and subsequent RGC death in adult eyes. $^{38}$ Retrograde transport of radiolabeled BDNF was impaired following IOP increase in rats, and accumulation of TrkB immunolabeling was found in the optic nerve head in this model. ${ }^{39}$ Therefore, supplementation therapy using neurotrophic factors is an attractive method for protection of RGCs and axons in glaucoma. We previously proposed a model in which exogenously applied or microglia-derived neurotrophins regulate photoreceptor survival indirectly by regulating secondary trophic factor production in Müller cells. ${ }^{31}$ However, the effects that such a glia-neuron interaction would have in vivo or whether it would be relevant for the protection of neural cell types that express TrkB remained unclear. To explore this point, we prepared two conditional KO mice in which TrkB was deleted from Müller glia (TrkB GFAP KO mice) or from two types of retinal neurons (RGCs and amacrine cells). ${ }^{24}$ Surprisingly, the extent of glutamate-induced retinal degeneration was similar in these two mutant mice. Furthermore, BDNF failed to increase the production of BDNF, bFGF, CNTF and GDNF in cultured Müller cells prepared from TrkB GFAP KO mice. These results suggest a possibility that BMD-induced activation of BDNF signaling in glia is critically involved in RGC protection. One interesting point is that BMD increased NGF production in Müller cells. A recent study reported that NGF eye drops reduced $R G C$ loss in patients with advanced glaucoma, and indicated long-lasting improvements in visual field, optic nerve function, contrast sensitivity and visual acuity. ${ }^{40}$ However, several studies have failed to observe NGF-mediated RGC survival, ${ }^{41,42}$ thus further studies are required before topical NGF can be considered for application in human glaucoma. Based on our findings, IOP-independent neuroprotection by BMD may be partly explained by BMD-induced upregulation 

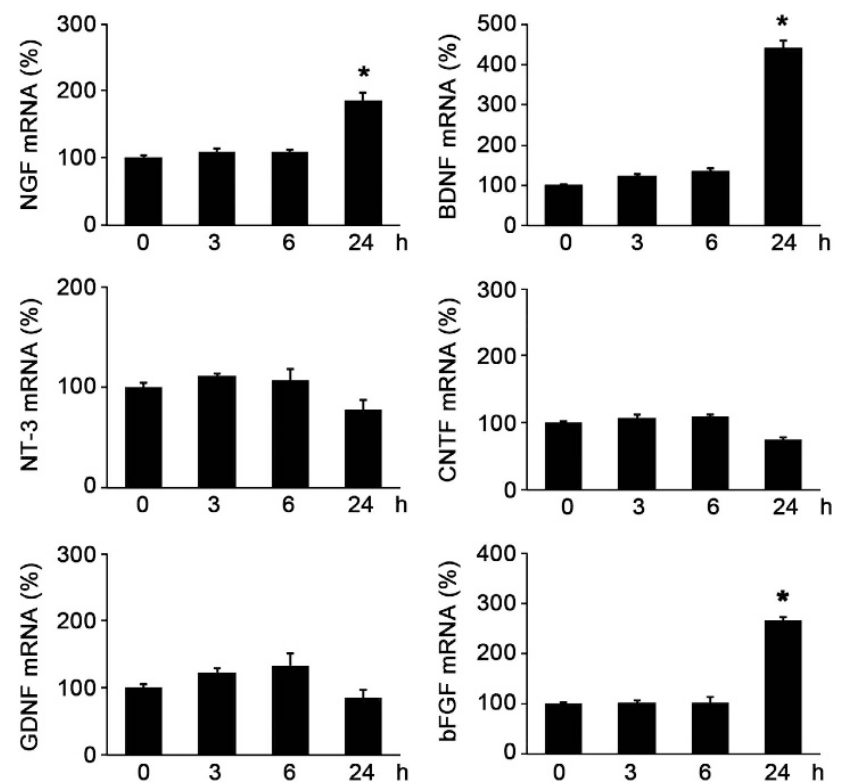

Figure 6 Effects of BMD on trophic factor productions in cultured Müller cells. mRNA expression levels of NGF, BDNF, NT-3, CNTF, GDNF and bFGF were determined using quantitative real-time PCR. Glyceraldehyde 3-phosphate dehydrogenase (GAPDH) was used as an internal control. Each mRNA production level before stimulation $(0 \mathrm{~h})$ was estimated as $100 \%$. The data are presented as means \pm S.E.M. of six samples for each experiment. ${ }^{*} P<0.01$

of trophic factors in surrounding glial cells. However, another important point to consider is that Müller cells may exert both neuroprotective and detrimental effects during retinal degeneration. ${ }^{10,26,30,31,37}$ In animal models of high IOP, BMD treatment suppressed the GFAP immunoreactivity compared with the animals after treatment with timolol. ${ }^{43,44}$ The mechanism by which BMD influences GFAP immunoreactivity is not well understood, but BMD may afford greater neuroprotection by attenuating the retinal glial reaction. We cannot directly compare these results with our present results from NTG model mice, but future studies examining the effects of gliosis on trophic factor productions in Müller cells may be interesting.

In summary, we showed that BMD prevents RGC death and reduces a degree of visual impairment in a mouse model of NTG by suppressing excitotoxicity in RGCs and stimulating trophic factor release from Müller cells, indicating that BMD is capable of activating several pathways in both neural and glial cells. There has been considerable progress in characterization of molecular pathways that regulate RGC survival. To this end, therapeutic potentials of several neurotrophic factors in protecting RGCs are emerging and some clinical trials for retinal diseases are underway. ${ }^{45,46}$ Therefore, it is important to establish the long-term effects of BMD, and to determine whether BMD can synergistically stimulate RGC survival in combination with currently available therapy in glaucomatous eyes.

\footnotetext{
Materials and Methods

Mice. Experiments were performed using EAAC1 KO mice (Miltenyi Biotec $\mathrm{GmbH}$, Bergisch Gladbach, Germany) ${ }^{3,8,10}$ in accordance with the Tokyo Metropolitan Institute of Medical Science Guidelines for the Care and Use of Animals.
}

Drug administration. Mice were treated with $5 \mu \mathrm{l}$ drops of $1.0 \%$ BMD tartrate (Senju Pharmaceutical Co., Ltd, Osaka, Japan) dissolved in PBS every day from $5 \mathrm{~W}$ to $8 \mathrm{~W}$. PBS was administered as a control.

Histologic and morphometric studies. Paraffin-embedded retinal sections of $7 \mu \mathrm{m}$ thickness were cut through the optic nerve and stained with hematoxylin and eosin (H\&E). The RGC number and the extent of retinal degeneration were quantified in two ways. ${ }^{9}$ First, the thickness of the IRL (between the internal limiting membrane and the interface of the outer plexiform layer and the outer nuclear layer) was analyzed. Second, in the same sections, the number of neurons in the GCL was counted from one ora serrata through the optic nerve to the other ora serrata.

Imaging acquisition of spectral-domain OCT. Spectral-domain OCT (SD-OCT) (RS-3000; Nidek, Aichi, Japan) examinations were performed at 5W and $8 \mathrm{~W}$. For fundus imaging, polymethyl methacrylate contact lenses optimal for mice (UNICON, Osaka, Japan) were placed on the corneas. Use of the lenses prevents anesthesia-induced cataract progression. A 60-D adaptor lens was placed on the objective lens of the Multiline OCT to focus on the mouse retina. All the images were location matched, scanning vertically through the center of the optic nerve head at 3 disc diameter lengths above the optic nerve head. ${ }^{10,26}$ The average thickness of GCC (between the internal limiting membrane and the interface of the inner plexiform layer and the inner nuclear layer) was measured. In this study, the maximum number of B-scans set by the manufacturer (50 for line scans) was used for averaging.

mfERG. Mice at $5 \mathrm{~W}$ and $8 \mathrm{~W}$ were anesthetized by intraperitoneal injection of sodium pentobarbital. The pupils were dilated with $0.5 \%$ phenylephrine hydrochloride and $0.5 \%$ tropicamide. mfERGs were recorded using a VERIS 6.0 system (Electro-Diagnostic Imaging, Redwood City, CA, USA). The visual stimulus consisted of seven hexagonal areas scaled with eccentricity. The stimulus array was displayed on a high-resolution black and white monitor driven at a frame rate of $100 \mathrm{~Hz}$. The second-order kernel, which is impaired in patients with glaucoma, was analyzed. ${ }^{3,9,28}$

IOP measurement. IOP was measured by a commercial rebound tonometer (TonoLab; Colonial Medical Supply, Franconia, NH, USA) in anesthetized mice as reported previously. ${ }^{9,10}$ To minimize variation, the data were collected during a time window of 4-6 min after injection of the anesthetic, during which IOP plateaus. IOP was measured at $5 \mathrm{~W}$ and $8 \mathrm{~W}$. As the $24 \mathrm{~h}$ IOP pattern in mouse eyes is biphasic, with IOP being the highest at around 2100 hours, ${ }^{29}$ we examined IOP between 2000 and 2300 hours.

Immunoblot analysis. Immunoblotting was performed as reported previously. ${ }^{5}$ Membranes were incubated with an antibody against NR2B (1:1000; BD Biosciences, San Jose, CA, USA), or phosphorylated NR2B (1:300; BD Biosciences) or actin (1:1000; BD Biosciences).

Immunohistochemistry. Retinas were examined by immunostaining as reported previously. ${ }^{5,9,10}$ Immunohistochemistry was performed using the antibody for phosphorylated NR2B (1:1000; BD Biosciences). Quantitative analysis of the stained region was carried out using BZ-H1C (Keyence Software, Osaka, Japan).

Cell culture and quantitative real-time PCR. Primary Müller cells $^{10,24,30,31}$ derived from WT mice were stimulated with BMD $(1 \mu \mathrm{M})$ for 3,6 , 12 and $24 \mathrm{~h}$, and processed for quantitative PCR analysis. Quantitative RT-PCR was performed using the ABI 7300 Real-Time PCR system (Applied Biosystems, Foster City, CA, USA) with Power SYBR Green PCR Master Mix (Applied Biosystems) as reported previously. ${ }^{47}$ Complementary DNA reverse-transcribed from total RNA was amplified using primers specific for NGF (sense: $5^{\prime}$-CGAC TCCAAACACTGGAACTCA-3'; antisense: $5^{\prime}$-GCCTGCTTCTCATCTGTTGTCA- $3^{\prime}$ ), BDNF (sense: 5'-ATGCCGCAAACATGTCTATGAG-3'; antisense: 5'-TGACCCA CTCGCTAATACTGTCA-3'), NT-3 (sense: 5'-GTTCCAGCCAATGATTGCAA-3'; antisense: 5'-GGGCGAATTGTAGCGTCTCT-3'), CNTF (sense: $5^{\prime}$-GGTGACTTC CATCAGGCAATACA-3'; antisense: 5'-CTGTTCCAGAAGCGCCATTAAC-3'), GDNF (sense; 5'-GGCCTACCTTGTCACTTGTTAGC-3'; antisense: 5'-GGCCTAC TTTGTCACTTGTTAGC-3'), bFGF (sense: 5'-CACCAGGCCACTTCAAGGA-3'; antisense: $5^{\prime}$-GATGGATGCGCAGGAAGAA-3') and GAPDH (sense: $5^{\prime}$-TGCACC ACCAACTGCTTAG-3'; antisense: 5'-GGATGCAGGGATGATGTTC-3'). 
Statistics. For statistical comparison of two samples, we used a two-tailed Student's $t$-test. Data are presented as means \pm S.E.M. $P<0.05$ was regarded as statistically significant.

\section{Conflict of Interest}

The authors declare no conflict of interest.

Acknowledgements. We thank X Guo, D Kittaka, M Kunitomo and K Okabe for their invaluable technical support. This study was supported by the Ministry of Education, Culture, Sports, Science and Technology of Japan (KN, AK, CH, YM and TH), Senju Pharmaceutical Co. Ltd and the Funding Program for Next Generation World-Leading Researchers (NEXT Program) (TH).

1. Quigley HA, Broman AT. The number of people with glaucoma worldwide in 2010 and 2020. Br J Ophthalmol 2006; 90: 262-267.

2. Iwase A, Suzuki Y, Araie M, Yamamoto $T$, Abe H, Shirato $S$ et al. The prevalence of primary open-angle glaucoma in Japanese: the Tajimi Study. Ophthalmology 2004; 111: 1641-1648.

3. Harada T, Harada C, Nakamura K, Quah HA, Okumura A, Namekata K et al. The potential role of glutamate transporters in the pathogenesis of normal tension glaucoma. J Clin Invest 2007; 117: 1763-1770.

4. Desai PV, Caprioli J. The treatment of normal-tension glaucoma. Prog Brain Res 2008; 173: $195-210$.

5. Namekata K, Kimura A, Kawamura K, Guo X, Harada C, Tanaka K et al. Dock3 attenuates neural cell death due to NMDA neurotoxicity and oxidative stress in a mouse model of normal tension glaucoma. Cell Death Differ 2013; 20: 1250-1256.

6. Danbolt NC. Glutamate uptake. Prog Neurobiol 2001; 65: 1-105.

7. Harada T, Harada C, Watanabe M, Inoue Y, Sakagawa T, Nakayama N et al. Functions of the two glutamate transporters GLAST and GLT-1 in the retina. Proc Natl Acad Sci USA 1998; 95: 4663-4666.

8. Namekata K, Harada C, Guo X, Kikushima K, Kimura A, Fuse N et al. Interleukin-1 attenuates normal tension glaucoma-like retinal degeneration in EAAC1-deficient mice. Neurosci Lett 2009; 465: 160-164.

9. Harada C, Namekata K, Guo X, Yoshida H, Mitamura Y, Matsumoto $Y$ et al. ASK1 deficiency attenuates neural cell death in GLAST-deficient mice, a model of normal tension glaucoma. Cell Death Differ 2010; 17: 1751-1759.

10. Semba K, Namekata K, Guo X, Harada C, Harada T, Mitamura Y. Renin-angiotensin system regulates neurodegeneration in a mouse model of normal tension glaucoma. Cell Death Dis (in press).

11. Osborne NN. Pathogenesis of ganglion 'cell death' in glaucoma and neuroprotection: focus on ganglion cell axonal mitochondria. Prog Brain Res 2008; 173: 339-352.

12. Plafker SM. Oxidative stress and the ubiquitin proteolytic system in age-related macular degeneration. Adv Exp Med Biol 2010; 664: 447-456.

13. Naskar R, Vorwerk CK, Dreyer EB. Concurrent downregulation of a glutamate transporter and receptor in glaucoma. Invest Ophthalmol Vis Sci 2000; 41: 1940-1944.

14. Akman A, Cetinkaya A, Akova YA, Ertan A. Comparison of additional intraocular pressurelowering effects of latanoprost vs brimonidine in primary open-angle glaucoma patients with intraocular pressure uncontrolled by timolol-dorzolamide combination. Eye 2005; 19: 145-151.

15. Metoki T, Ohguro H, Ohguro I, Mamiya K, Ito T, Nakazawa M. Study of effects of antiglaucoma eye drops on $\mathrm{N}$-methyl-D-aspartate-induced retinal damage. Jpn J Ophthalmol 2005; 49: 453-461.

16. Lee KY, Nakayama M, Aihara M, Chen YN, Araie M. Brimonidine is neuroprotective against glutamate-induced neurotoxicity, oxidative stress, and hypoxia in purified rat retinal ganglion cells. Mol Vis 2010; 16: 246-251.

17. Lee D, Kim KY, Noh YH, Chai S, Lindsey JD, Ellisman MH et al. Brimonidine blocks glutamate excitotoxicity-induced oxidative stress and preserves mitochondrial transcription factor a in ischemic retinal injury. PLoS One 2012; 7: e47098.

18. Dong CJ, Guo Y, Agey P, Wheeler L, Hare WA. Alpha2 adrenergic modulation of NMDA receptor function as a major mechanism of RGC protection in experimental glaucoma and retinal excitotoxicity. Invest Ophthalmol Vis Sci 2008; 49: 4515-4522.

19. Levkovitch-Verbin H, Harris-Cerruti C, Groner Y, Wheeler LA, Schwartz M, Yoles E. RGC death in mice after optic nerve crush injury: oxidative stress and neuroprotection. Invest Ophthalmol Vis Sci 2000; 41: 4169-4174.

20. Gao H, Qiao X, Cantor LB, WuDunn D. Up-regulation of brain-derived neurotrophic factor expression by brimonidine in rat retinal ganglion cells. Arch Ophthalmol 2002; 120: 797-803.

21. Wen R, Cheng T, Li Y, Cao W, Steinberg RH. Alpha 2-adrenergic agonists induce basic fibroblast growth factor expression in photoreceptors in vivo and ameliorate light damage. J Neurosci 1996; 16: 5986-5992.

22. Lai RK, Chun T, Hasson D, Lee S, Mehrbod F, Wheeler L. Alpha-2 adrenoceptor agonist protects retinal function after acute retinal ischemic injury in the rat. Vis Neurosci 2002; 19: 175-185.

23. Fujita $Y$, Sato A, Yamashita T. Brimonidine promotes axon growth after optic nerve injury through Erk phosphorylation. Cell Death Dis 2013; 4: e763.
24. Harada C, Guo X, Namekata K, Kimura A, Nakamura K, Tanaka K et al. Glia- and neuron-specific functions of TrkB signalling during retinal degeneration and regeneration. Nat Commun 2011; 2: 189

25. Peng M, Li Y, Luo Z, Liu C, Laties AM, Wen R. Alpha2-adrenergic agonists selectively activate extracellular signal-regulated kinases in Müller cells in vivo. Invest Ophthalmol Vis Sci 1998; 39: 1721-1726.

26. Katome T, Namekata K, Guo X, Semba K, Kittaka D, Kawamura K et al. Inhibition of ASK1-p38 pathway prevents neural cell death following optic nerve injury. Cell Death Differ 2013; 20: 270-280.

27. Hasegawa S, Ohshima A, Hayakawa Y, Takagi M, Abe H. Multifocal electroretinograms in patients with branch retinal artery occlusion. Invest Ophthalmol Vis Sci 2013; 2: 298-304.

28. Sutter EE, Bearse Jr MA. The optic nerve head component of the human ERG. Vision Res 1999; 39: 419-436.

29. Aihara M, Lindsey JD, Weinreb RN. Twenty-four-hour pattern of mouse intraocular pressure. Exp Eye Res 2003; 77: 681-686.

30. Harada T, Harada C, Nakayama N, Okuyama S, Yoshida K, Kohsaka S et al. Modification of glial-neuronal cell interactions prevents photoreceptor apoptosis during light-induced retinal degeneration. Neuron 2000; 26: 533-541

31. Harada T, Harada C, Kohsaka S, Wada E, Yoshida K, Ohno S et al. Microglia-Müller glia cell interactions control neurotrophic factor production during light-induced retinal degeneration. J Neurosci 2002; 22: 9228-9236.

32. van Velthoven ME, Faber DJ, Verbraak FD, van Leeuwen TG, de Smet MD. Recent developments in optical coherence tomography for imaging the retina. Prog Retin Eye Res 2007; 26: 57-77.

33. Krupin T, Liebmann JM, Greenfield DS, Ritch R, Gardiner S. Low-Pressure Glaucoma Study Group. A randomized trial of brimonidine versus timolol in presenving visual function: results from the Low-Pressure Glaucoma Treatment Study. Am J Ophthalmol 2011; 151: 671-681.

34. Zhang K, Zhang L, Weinerb RN. Ophthalmic drug discovery: novel targets and mechanisms for retinal diseases and glaucoma. Nat Rev Drug Discov 2012; 11: 541-559.

35. Christie MJ, Napier IA, Eckert A, Staufenbiel M, Hardeman E, Götz J. Dendritic function of tau mediates amyloid- $\beta$ toxicity in Alzheimer's disease mouse models. Cell 2010; 142: 387-397.

36. Milnerwood AJ, Gladding CM, Pouladi MA, Kaufman AM, Hines RM, Boyd JD et al. Early increase in extrasynaptic NMDA receptor signaling and expression contributes to phenotype onset in Huntington's disease mice. Neuron 2010; 65: 178-190.

37. Bringmann A, landiev I, Pannicke T, Wurm A, Hollborn M, Wiedemann P et al. Cellular signaling and factors involved in Müller cell gliosis: neuroprotective and detrimental effects. Prog Retin Eye Res 2009; 28: 423-451.

38. Knox DL, Eagle Jr RC, Green WR. Optic nerve hydropic axonal degeneration and blocked retrograde axoplasmic transport: histopathologic features in human high-pressure secondary glaucoma. Arch Ophthalmol 2007; 125: 347-353.

39. Pease ME, McKinnon SJ, Quigley HA, Kerrigan-Baumrind LA, Zack DJ. Obstructed axonal transport of BDNF and its receptor TrkB in experimental glaucoma. Invest Ophthalmol Vis Sci 2000; 41: 764-774.

40. Lambiase A, Aloe L, Centofanti M, Parisi V, Mantelli F, Colafrancesco V et al. Experimental and clinical evidence of neuroprotection by nerve growth factor eye drops: Implications for glaucoma. Proc Natl Acad Sci USA 2009; 106: 13469-13474.

41. Bai Y, Dergham P, Nedev H, Xu J, Galan A, Rivera JC et al. Chronic and acute models of retinal neurodegeneration TrkA activity are neuroprotective whereas p75NTR activity is neurotoxic through a paracrine mechanism. J Biol Chem 2010; 285: 39392-39400.

42. Shi Z, Birman E, Saragovi HU. Neurotrophic rationale in glaucoma: a TrkA agonist, but not NGF or a p75 antagonist, protects retinal ganglion cells in vivo. Dev Neurobiol 2007; 67: 884-894.

43. WoldeMussie E, Ruiz G, Wijono M, Wheeler LA. Neuroprotection of retinal ganglion cells by brimonidine in rats with laser-induced chronic ocular hypertension. Invest Ophthalmol Vis Sci 2001; 42: 2849-2855.

44. Vidal L, Díaz F, Villena A, Moreno M, Campos JG, Pérez de Vargas I. Reaction of Müller cells in an experimental rat model of increased intraocular pressure following timolol, latanoprost and brimonidine. Brain Res Bull 2010; 82: 18-24.

45. Tombran-Tink J. PEDF in angiogenic eye diseases. Curr Mol Med 2010; 10: 267-278.

46. Kolomeyer AM, Zarbin MA. Trophic factors in the pathogenesis and therapy for retinal degenerative diseases. Surv Ophthalmol 2014; 59: 134-165.

47. Guo X, Nakamura K, Kohyama K, Harada C, Behanna HA, Watterson DM et al. Inhibition of glial cell activation ameliorates the severity of experimental autoimmune encephalomyelitis. Neurosci Res 2007; 59: 457-466.

Cell Death and Disease is an open-access journal published by Nature Publishing Group. This work is licensed under a Creative Commons Attribution 3.0 Unported License. The images or other third party material in this article are included in the article's Creative Commons license, unless indicated otherwise in the credit line; if the material is not included under the Creative Commons license, users will need to obtain permission from the license holder to reproduce the material. To view a copy of this license, visit http://creativecommons.org/licenses/by/3.0/ 